\title{
High sensitivity photonic crystal pressure sensor
}

\section{Biallo}

\section{De Sario}

\section{A. D'Orazio dorazio@poliba.it}

\section{Marrocco}

\section{Petruzzelli}

\section{A. Vincenti \\ F. Prudenzano}

\section{T. Stomeo}

\section{M.Grande}

G. Visimberga

\section{R.Cingolani}

\section{De Vittorio}

Dipartimento di Elettrotecnica ed Elettronica, Politecnico di Bari, Via Orabona 4, 70125 Bari, Italy

Dipartimento di Elettrotecnica ed Elettronica, Politecnico di Bari, Via Orabona 4, 70125 Bari, Italy

Dipartimento di Elettrotecnica ed Elettronica, Politecnico di Bari, Via Orabona 4, 70125 Bari, Italy

Dipartimento di Elettrotecnica ed Elettronica, Politecnico di Bari, Via Orabona 4, 70125 Bari, Italy

Dipartimento di Elettrotecnica ed Elettronica, Politecnico di Bari, Via Orabona 4, 70125 Bari, Italy

Dipartimento di Elettrotecnica ed Elettronica, Politecnico di Bari, Via Orabona 4, 70125 Bari, Italy

Dipartimento di Ingegneria dell'Ambiente e per lo Sviluppo Sostenibile, Politecnico di Bari - II Facoltà di Ingegneria, Viale del Turismo 8, 74100 Taranto, Italy

National Nanotechnology Laboratory (NNL) - CNR-INFM, Università di Lecce, Via Arnesano, 73100, Lecce, Italy

National Nanotechnology Laboratory (NNL) - CNR-INFM, Università di Lecce, Via Arnesano, 73100, Lecce, Italy

National Nanotechnology Laboratory (NNL) - CNR-INFM, Università di Lecce, Via Arnesano, 73100, Lecce, Italy

National Nanotechnology Laboratory (NNL) - CNR-INFM, Università di Lecce, Via Arnesano, 73100, Lecce, Italy

National Nanotechnology Laboratory (NNL) - CNR-INFM, Università di Lecce, Via Arnesano, 73100, Lecce, Italy

A two-dimensional photonic crystal microcavity coupled to a photonic crystal waveguide is proposed to realise a high sensitive pressure sensor, designed on a GaAs membrane. A theoretical model is developed to evaluate the change of the refractive index induced by the application of a force onto a sensing surface. A linear calibration curve is obtained relating the resonant drop position to the applied pressure. [DOI: 10.2971/jeos.2007.07017]

Keywords: Integrated optics, optical waveguide, sensor

\section{INTRODUCTION}

The photonic crystal capability to control the flow of the light makes this kind of structures very attractive for several applications in the field of integrated optics and telecommunications $[1,2]$. However in the last years a large number of investigations has shown the potential improvement consequent to the introduction of photonic crystal technology in the wide field of optical sensors. In fact the compact dimensions and the high sensitivity of the optical properties with respect to the background variables are essential qualities for the implementation of an optical sensor.

An example of sensing photonic crystal device recently proposed is the ultracompact biochemical sensor [3], built with two-dimensional photonic crystal microcavity, fabricated on silicon on insulator substrate, capable to detect the change of the refractive index, equal to 0.002 , by measuring the resonant wavelength of the microcavity.

Another suggestive application is the micro-displacement optical sensor proposed in [4,5] which can be integrated in a micro-electro-mechanical system. A very sensitive displacement sensor has been realised by considering a twodimensional photonic crystal waveguide, assisted by a fixed and a mobile segments. The measure of the displacement can be accomplished evaluating the variation of the intensity of the guided modes with respect to the relative position of the two segments.

A smart device, fabricated using a multi-layer, metal dielectric PBG structure with electrically controllable optical properties was proposed in [6]: it can be used for sensing applications, in 
harsh or hostile environments, as pressure sensor, micro gradiometre or micro accelerometer.

Exploiting the guided resonance phenomenon, it is possible to fabricate structures that are highly sensitive to longitudinal displacements, for example by using the photonic crystal slabs as mirrors to form Fabry Perot cavity [7]. In this case, the highest achievable sensitivity is directly related to the maximum reflectivity expected in a single slab.

In this paper we report the full design of a photonic crystal pressure sensor constituted by a microcavity coupled to a photonic crystal waveguide designed in a GaAs membrane. The sensor operation is based on the detection of the frequency shift of the localised state supported by the microcavity due to the variation of the refractive index of the GaAs induced by the pressure application. The sensor sensitivity improves as the quality factor of the microcavity increases $[8,9]$, while the simultaneous presence of a line defect and a point defect allows an easier detection of the shift in the frequency drop position [10].

\section{THEORETICAL MODEL}

The sensor is realised in a two-dimensional photonic crystal GaAs membrane, having a 600nm thick GaAs layer. The sketch of the whole device is shown in Figure 1. The photonic crystal is composed by a triangular lattice of air holes, characterised by a lattice constant equal to $360 \mathrm{~nm}$, an air-hole radius equal to $0.35 \mathrm{a}$, whereas the hole-depth is equal to $600 \mathrm{~nm}$. A line defect is introduced by removing a row of air holes in the $\Gamma K$ direction (W1), allowing guided modes to propagate within the photonic band gap. The W1 is coupled to a resonant cavity: the transmission spectrum experiences the presence of a frequency drop caused by the presence of the cavity, which is able to trap the light at a specific frequency.

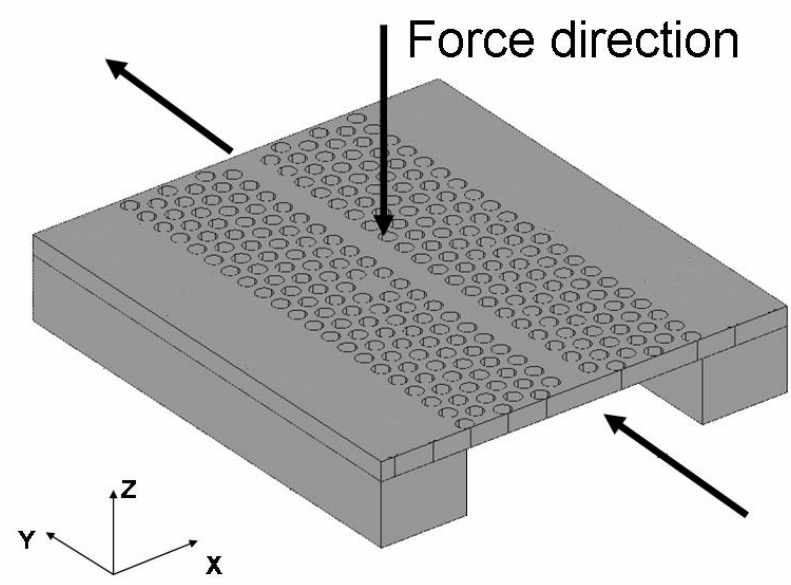

FIG. 1 Sketch of the Photonic crystal membrane sensor.

The sensing operation principle is based on the change of the refractive index induced by the photoelastic, piezoelectric and electrooptic effects of the membrane material, i.e. GaAs, which must be considered when a force is applied on the device sur- face. The change modifies the transmission spectrum of the photonic crystal membrane with respect to the unperturbed PC, and in particular, induces a frequency shift of the localised resonant state (i.e. shift of the frequency drop).

The relationship between the applied pressure and the refractive index change is expressed in terms of the variation of the dielectric impermeability tensor $\mathbf{b}$ (the inverse of the dielectric constant tensor $\epsilon$ ) by the following relation:

$$
\Delta \mathbf{b}=\left(\mathbf{p}^{E}: \mathbf{s}-\mathbf{r}^{T}: \mathbf{g}^{T}\right): \mathbf{T}
$$

where $\mathbf{p}$ is the photoelastic fourth-order tensor at constant electric field, $\mathbf{s}$ is the fourth-rank elastic compliance sensor, $\mathbf{r}^{T}=\mathbf{r}^{S}+\mathbf{p}: \mathbf{d}^{S}$ is the electrooptic third-rank tensor at constant stress in which $\mathbf{r}^{S}$ is the third-order electrooptic tensor at constant strain $\mathbf{u}$ and $\mathbf{d}^{S}$ is the third-rank piezoelectric tensor at constant strain, $\mathbf{g}^{T}$ is the third-rank piezoelectric tensor at constant stress, and the symbol : means double contraction $[11,12]$.

Assuming $\mathbf{q}=\left(\mathbf{p}^{E}: \mathbf{s}-\mathbf{r}^{T}: \mathbf{g}^{T}\right)$, the values of each element of the tensor $\mathbf{q}$ are $q_{11}=q_{22}=q_{33}=-0.1338 \cdot 10^{-11} \mathrm{~m}^{2} / \mathrm{N}$, $q_{44}=q_{55}=q_{66}=-0.4388 \cdot 10^{-11} \mathrm{~m}^{2} / \mathrm{N}, q_{12}=q_{21}=q_{23}=$ $q_{32}=q_{13}=q_{31}=-0.0978 \cdot 10^{-11} \mathrm{~m}^{2} / \mathrm{N}$, the other elements of the tensor $\mathbf{q}$ are equal to 0 .

Since we assume that the force direction is orthogonal to the photonic crystal plane (see Figure 1) and the coordinate axes coincide with the principal directions of the crystal, the stress tensor $\mathbf{T}$, considering the contracted form, displays only one element different from zero, $\mathbf{T}_{3}$.

The variation of the dielectric impermeability $\Delta \mathbf{b}$ can be arranged as $\Delta \mathbf{b}_{1}=\mathbf{q}_{12} \mathbf{T}_{3}, \Delta \mathbf{b}_{2}=\mathbf{q}_{12} \mathbf{T}_{3}, \Delta \mathbf{b}_{3}=\mathbf{q}_{11} \mathbf{T}_{3}, \Delta \mathbf{b}_{4}=$ $\Delta \mathbf{b}_{5}=\Delta \mathbf{b}_{6}=\mathbf{0}$.

The calculations related to the change of the impermeability tensor $\mathbf{b}$, accounting for the strain and stress effects acting on the lattice, evaluated using Eq. 1 and considering the contribution of all effects reveal that the main contribute to the dielectric impermeability tensor variation is due to the photoelastic effect.

In order to model exactly the action and the effects of the applied pressure on the membrane a structural strain, stress and displacement analysis has been performed through a commercial 3D finite element method code (COMSOL MultiphysicsFemlab). The mechanical analysis shows how the membrane bends when the pressure is applied uniformly along the direction orthogonal to the propagation plane and, at the same time, gives back the results concerning the distribution of the strain and the stress on the whole structure. Figure 2 depicts the effect of the applied stress tensor when a pressure of $2 \mathrm{GPa}$ is considered: the membrane bends along the longitudinal direction, but the cavity and the $\mathrm{W} 1$ waveguide remain almost at the same height. This effect does not influence the coupling capability to and from the sensor, as the bent angle becomes relevant at the edges of the structure. Figure 3 shows the maximum displacement experienced by the membrane as the pressure value is increased: the bending entity is proportional to the applied pressure and reaches the maximum value of about 
$0.8 \mu \mathrm{m}$ at the centre of the membrane when the pressure of 2GPa is applied.

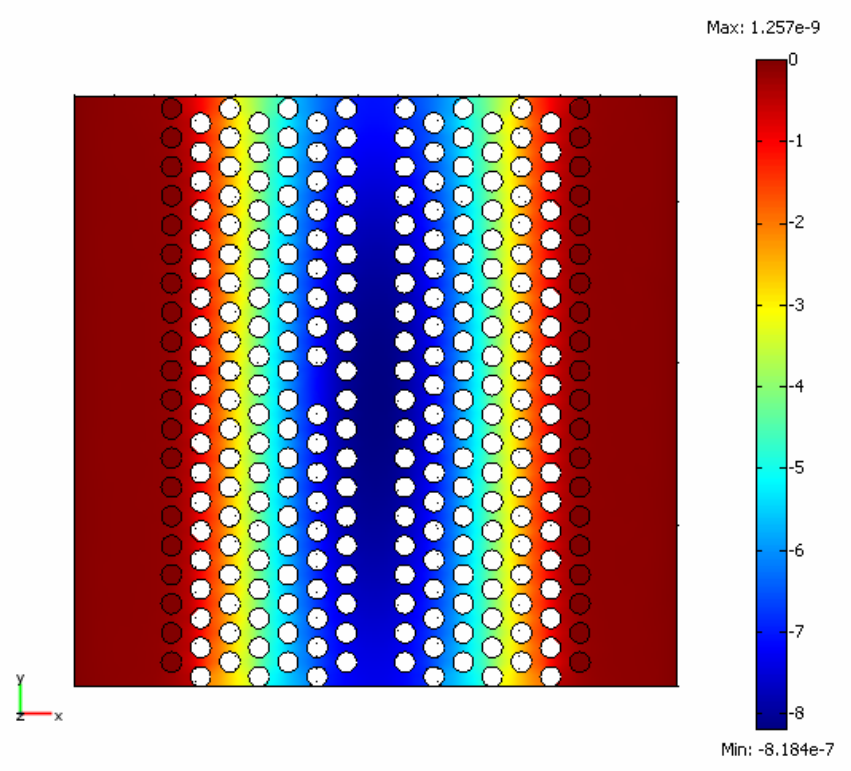

FIG. 2 Displacement distribution after the application of the force in the direction perpendicular to the propagation plane.

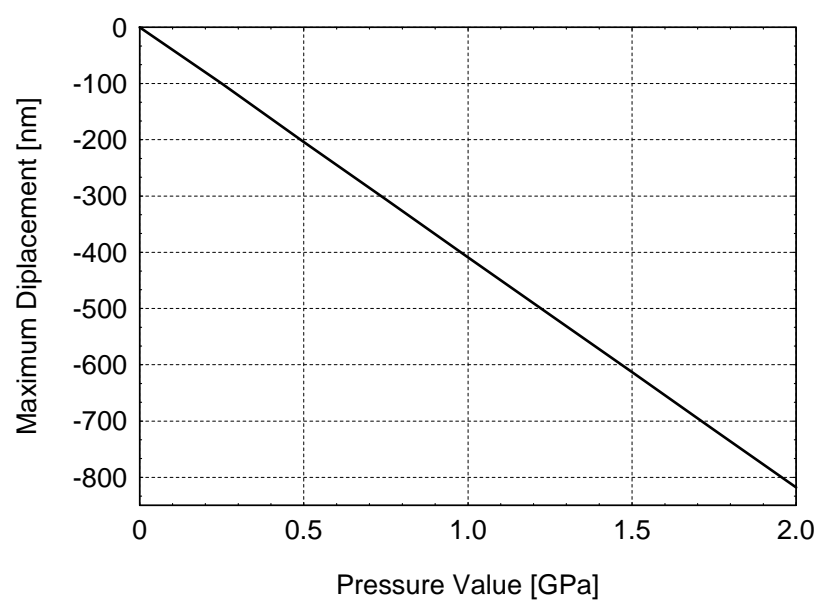

FIG. 3 Maximum displacement experimented.

Moreover, no breaking points for the membrane have been pointed out until this pressure value is considered. We also verified that if we consider applied pressure values beyond $2 \mathrm{GPa}$, the membrane bends critically, thus affecting the propagation within the membrane and consequently the whole functionality of the sensor.

The structural/strain analysis has shown that the distribution of the stress is homogeneous when the bases holding the whole structure have at least the size of one sixth of the entire dimension $x$ (see Figure 1), so that the refractive index variation can be considered uniform in the whole structure.

\section{NUMERICAL RESULTS}

When the pressure is applied onto the whole device, the GaAs becomes anisotropic, displaying different values of the refrac- tive index tensor elements depending on the applied force direction. For instance, if a pressure of 1GPa is applied uniformly onto the whole device, the GaAs refractive index tensor $[13,14]$ assumes the following values: $n_{11}=n_{22}=3.4274$, $n_{33}=3.4345$. As the Maxwell equations show, this GaAs anisotropy mainly affects the TM-like propagation, whereas our electromagnetic analysis concerns the TE-like modes.

The 3D-electromagnetic analysis of the sensor has been performed by means of a commercial code, i.e. RSoft-FullWave, based on a finite difference time domain algorithm (FDTD). Different 3D-electromagnetic calculations concerning TE-like mode propagation have been carried out. First, the isotropic GaAs membrane having refractive index equal to 3.4274 , corresponding to $1 \mathrm{GPa}$ of applied pressure, has been considered as a benchmark calculation. In the isotropic case the drop peak corresponding to a bipole-like resonance supported by the cavity is centred around $\lambda=1.3108 \mu \mathrm{m}$. Then a calculation considering the refractive index tensor taking into account the GaAs anisotropy under 1GPa of applied pressure has been performed. The 3D results highlight that in the anisotropic case the drop peak is centred around $\lambda=1.3105 \mu \mathrm{m}$. Moreover, the anisotropy is responsible of a red-shift of the whole transmission band, although the shape of the transmission curve is not significantly modified. In order to prove that the anisotropy effect doesn't affect at all the overall expected behaviour of the sensor, two more anisotropic calculations have been performed when $0.25 \mathrm{GPa}$ and $2 \mathrm{GPa}$ of applied pressure are considered. The results stress that as the refractive index varies under the application of the pressure, the transmission spectrum experiences a red-shift, keeping its shape unchanged. In particular, when a pressure value of $0.25 \mathrm{GPa}$, $1 \mathrm{GPa}$ and $2 \mathrm{GPa}$ are considered the drop peak is centred at $\lambda=1.2992 \mu \mathrm{m}, \lambda=1.3105 \mu \mathrm{m}$ and $\lambda=1.3122 \mu \mathrm{m}$ respectively. The $3 \mathrm{D}$ calculations witness a difference between the isotropic and anisotropic case, weighting mainly on the position of the drop peak, but the anisotropy does not seem to modify neither the propagation characteristics of the TE-like modes nor the symmetry of the resonant mode. Although these calculations are more specific and reliable, they are time and memory consuming. Therefore, in order to show the sensor operation, an approximation has been introduced using an effective 2D analysis.

Furthermore, the 3D analysis has also been used to evaluate the out-of-plane losses related to the membrane; the geometrical parameters set for the design are fixed in order to minimise this effect. In fact, we checked out that the modes propagating in the waveguide are below the light line in the wavelength range of our interest, meaning that the modes did not have out-of-plane losses, as it is confirmed also in literature [15, 16]. The 3D calculations have been also developed to evaluate the radiation losses induced by the presence of the cavity: we notice that the dipole-like mode exhibits about $-35 \mathrm{~dB}$ of power emitted in the in-plane orthogonal direction. Hence we can assume that the out-of-plane losses are negligible in our calculations.

Since our electromagnetic analysis involves the TE-like propagation only, on the basis of the previous considerations the $3 \mathrm{D}$ problem has been reduced to a $2 \mathrm{D}$ one, and the refractive 
index of the 2D structure has been assumed equal to the effective refractive index of the fundamental TE-like mode of the membrane. For the perturbed structure, by means of Eq. 1, the refractive indices have been calculated for each pressure value, and the electromagnetic analysis has been performed for each equivalent $2 \mathrm{D}$ structure characterised by the corresponding effective refractive indices.

The electromagnetic analysis of the 2D structure has shown a large band gap for TE polarisation (magnetic field component parallel to the hole axis) between $\lambda_{1}=1.014 \mu \mathrm{m}$ and $\lambda_{2}=1.44 \mu \mathrm{m}$, whereas a smaller stop band has been observed for TM polarisation. The wide TE-band gap of the GaAs membrane suggested us to use the variable resonance position as detectable parameter. Moreover, the sensor under investigation has to be characterised by high sensitivity, in order to detect small pressure variations; by introducing a point defect within the unperturbed structure one is able to observe a localised resonant state in the band gap. Therefore it is necessary to enhance the quality factor of the point defect resonance to achieve a great field confinement inside the defect in order to realise a narrow resonant peak in the transmission spectrum. Furthermore, the functionality of the device will be complete when an efficient way to excite the microcavity modes and extract the light is engineered. As shown in Fig.4 (inset), the microcavity has been designed by reducing the neighbouring holes of the point defect (white holes in Figure 4 inset) from $0.35 \mathrm{a}$ to a radius value of $0.34 \mathrm{a}$ and enlarging the other surrounding ones (black holes in Figure 4 inset) to a value equal to $0.38 \mathrm{a}$, in order to achieve a good field confinement inside the microcavity and an efficient coupling to the light feeding system.

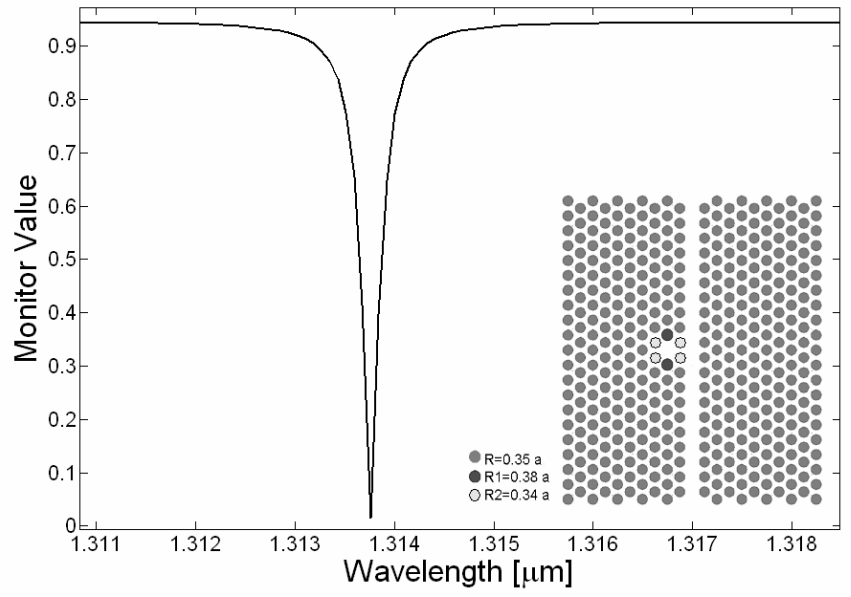

FIG. 4 Transmission spectrum showing the drop peak of the unperturbed structure. The inset depicts the microcavity coupled with the $W_{1}$ waveguide: black holes have radius $R 1=0.38 a$ and white holes have radius $R 2=0.34 a$ while the structure has radius $R=0.35 a$.

The simplest way of injecting the light into the cavity is to couple it with the fundamental mode of a W1 waveguide by evanescent field; this results in a frequency drop in the structure transmission spectrum (see Figure 4) [17, 18]. A trade-off between the obtainable values of the microcavity quality factor and the coupling efficiency of the cavity/W1 system becomes necessary to reach a balance between the high field con- finement and the fall-off of the transmission coefficient at the output of the waveguide.

The designed system is depicted in the inset of Figure 6: at $\lambda=1313,75 \mathrm{~nm}$ the microcavity supports a dipole-like mode characterised by a high quality factor $(Q=7000)$, which leads to a pronounced frequency drop that reaches value approaching to zero. The application of a pressure on the device results in an increment of the refractive index and consequently in a redshift of the drop position. The minimum shift experimented is $\Delta=0.17 \mathrm{~nm}$ (Figure 5), that is consistent with the resolution of an up-to-date commercial optical spectrum analyser.

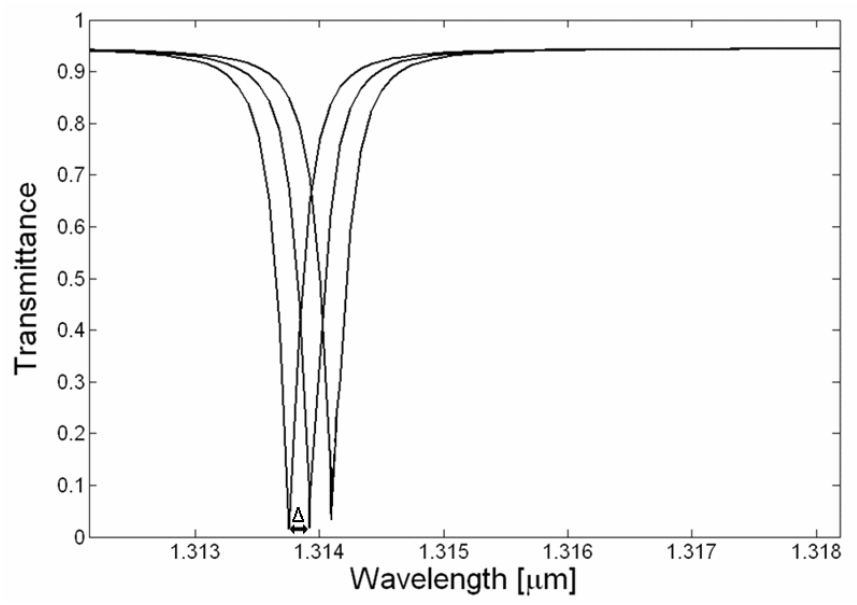

FIG. 5 Transmission spectra shows a minimum drop shift observed equal to $\Delta=$ $0.17 \mathrm{~nm}$

Therefore the minimum detectable pressure variation is $0.03 \mathrm{GPa}$; thus, if we consider an area of the pressure application equal to $48.5 \mu \mathrm{m}^{2}$, the numerical simulations show that the detectable minimum force is about $1.46 \mathrm{nN}$. This fact means that the device is able to discern different refractive indices with a precision of $\Delta n=0.00045$. Beyond this limit the numerical calculations show that the refractive index change is too small to cause a detectable frequency shift of the drop.

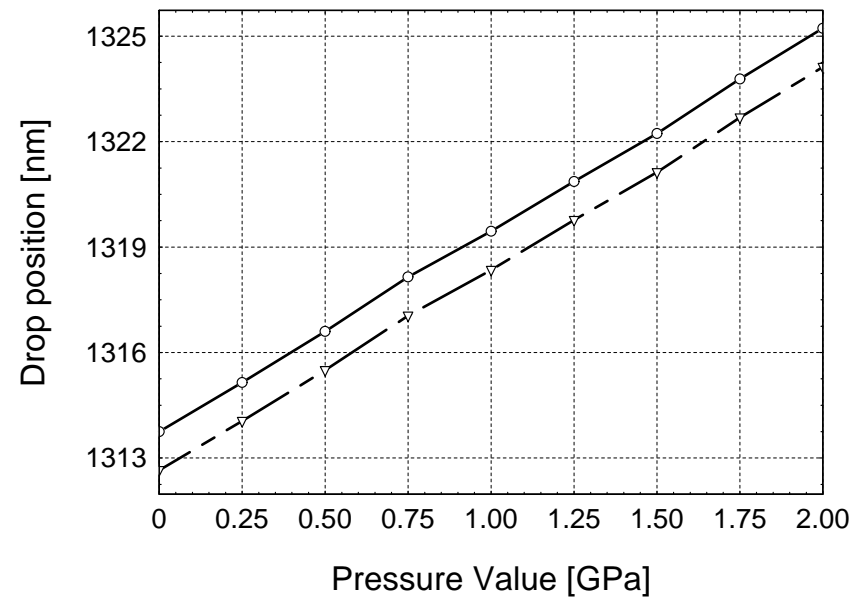

FIG. 6 Pressure sensor calibration curve: dispersion neglected (solid line) and dispersion included (dashed line). The drop peak experiences a blue-shift equal to $1.1 \mathrm{~nm}$ when the dispersion of the material is considered in the whole calculations 
The frequency drop positions moving towards higher wavelengths are evaluated for pressure values ranging from 0GPa to $2 \mathrm{GPa}$. By checking the frequency drop positions for different pressure values, it is possible to trace the sensor calibration curve as depicted in Figure 6 (solid line). It is clearly shown that the relationship between the drop position and the applied pressure follows a linear law, allowing linear measurements of the pressure. In this way we are able to estimate the sensor sensitivity $S$ evaluated in a unique fashion as the ratio between the drop peak position and the applied pressure variation, that is the slope of the calibration curve:

$$
S=\Delta \lambda / \Delta P
$$

The sensitivity value for the designed structure calculated by means of Eq. 2 is about $5.2 \mu \mathrm{m} / \mathrm{GPa}$. By considering a membrane structure, the sensitivity increases of about $10 \%$ respect to the value obtained with a photonic crystal GaAs/AlGaAs structure reported in [19].

All the aforementioned results are related to linear calculations, where the material dispersion is neglected. In order to estimate the effect of the material dispersion on the sensor functioning, we performed 2D calculations using both Lorentz and Drude models to mimic the real GaAs dispersion curve. The numerical results show that the introduction of the material dispersion is responsible of a blue-shift of the drop in the transmission spectrum. The drop shift remains constant as the value of applied pressure is incresed, since the dispersion curve is supposed to be unvaried under the application of the pressure and thus subjected to a red-shift as the refractive index values change. The calibration curve resulting by the dispersive calculations (dashed line in Figure 6) show that the relation between the drop position and the applied pressure is still linear.

We notice that the device sensitivity is linked to the intrinsic properties of the material the membrane is meant to be fabricated, while its resolution is strictly dependent on the quality factor of the microcavity that is, in turn, limited by the coupling efficiency to the waveguide.

\section{CONCLUSION}

We propose the design of a pressure photonic crystal sensor realised by considering a high-Q microcavity coupled to a W1 waveguide. The drop spectral position is the sensing parameter, being dependent on the refractive index of the membrane. Micrometric dimensions and high sensitivity make this structure eligible for sensing application in a wide range of application.

\section{ACKNOWLEDGEMENTS}

This work was sponsored by MiUR research contract, PRIN 2005: "Photonic Band Gap Nanosensors".

\section{References}

[1] J. D. Joannopoulos, R. D. Meade, J. N. Winn, Photonic Crystals Molding the flow of light (Princeton University press, 1995).

[2] E. Yablonovitch, "Photonic Band Gap Structures" J. Opt. Soc. Am. B 10, 283-295 (1993).

[3] E. Chow, A. Grot, L. W. Mirkarimi, M. Sigalas, G. Girolami, “Ultracompact biochemical sensor built with two-dimensional photonic crystal microcavity" Optics Lett. 29, 1093-1095 (2004).

[4] 0. Levy, B. Z. Steinberg, M. Nathan and A. Boag, "Ultrasensitive displacement sensing using photonic crystal waveguides" Appl. Phys. Lett. 86, 104102 (2005).

[5] Z. Xu, L. Cao, C. Gu, Q. He and G. Jin, "Micro displacement sensor based on line-defect resonant cavity in photonic crystal" Opt. Express 14, 298-305 (2006).

[6] S. Baglio, M. Bloemer, N. Savalli, M. Scalora "Development of novel optoelectromechanical systems based on "Transparent metals" PBG structures", IEEE Sens. J. 1, 288-295 (2001).

[7] W. Suh, M. F. Yanik, 0. Solgaard and S. Fan, "Displacementsensitive photonic crystal structures based on guided resonance in photonic crystal slabs" Appl. Phys. Lett. 82, 1999-2001 (2003).

[8] Y. Akahane, T. Asano, B. Song and S. Noda, "Fine-tuned high-Q photonic-crystal nanocavity" Opt. Express 13, 1202-1214 (2005).

[9] T. Yoshie, J. Vuckovic, A. Sherer, H. Chen and D. Deppe, "High quality two-dimensional photonic crystal slab cavities" App. Phys. Lett. 79, 4289-4291 (2001).

[10] Y. Akahane, T. Asano, H. Takano, B. Song, Y. Takana, S. Noda, “Twodimensional photonic-crystal-slab channel-drop filter with flat top response" Opt. Express 13, 2512-2530 (2005).

[11] I. P. Kaminow, An Introduction to Electrooptic Device (Academic Press, 1974).

[12] J. F. Nye, Physical Properties of Crystal (Oxford Science Publications, 1955).

[13] E. D. Palik, Handbook of Optical Constants of Solids (Academic Press, 1991).

[14] D. T. F. Marple, "Refractive index of GaAs" J. App. Phys. 35, 12411242 (1964).

[15] M. J. Cryan, D. C. Wong, I. J. Craddock, S. Yu, J. Rorison and C. J. Railton, "Calculation of losses in 2-D photonic crystal membrane waveguides using 3D-FDTD method", IEEE Photinic. Tech. L. 17, 58-60 (2005).

[16] Y. Sugimoto, Y. Tanaka, N. Ikeda, Y. Nakamura, K. Asakawa, “Low propagation loss of $0.76 \mathrm{~dB} / \mathrm{mm}$ in GaAs-based single line-defect two-dimensional photonic crystal slab waveguides up to $1 \mathrm{~cm}$ in length" Opt. Express 12, 1090-1096 (2004).

[17] B. Min, J. Min, H. Y. Park, "High efficiency surface emitting channel drop filters in two-dimensional photonic crystal slabs" Appl. Phys. Lett. 86, 011106 (2004).

[18] M. Imada, S. Noda, A. Chutinan, M. Mochizuki, T. Tamaka, "Channel drop filter using a single defect in a 2-D photonic crystal slab waveguide" J. Lightwave Technol. 20, 873-878 (2002).

[19] D. Biallo, A. D’Orazio, M. De Sario, V. Marrocco, V. Petruzzelli, M. A. Vincenti, F. Prudenzano, T. Stomeo, M. Grande, G. Visimberga, R. Cingolani, M. De Vittorio, "Design of a Photonic Crystal Microcavity Pressure Sensor" EOS Topical Meeting on Nanophotonics, Metamaterials and Optical Microcavities TOM 3, 187-188 (2006). 